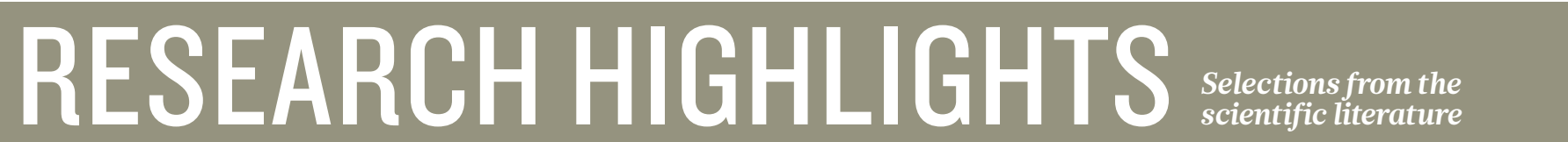

CANCER

\section{Weakening tumour defences}

Blocking just one enzyme could make some tumours easier to kill with radiation therapy.

Fei-Fei Liu at the University of Toronto in Ontario, Canada, and her team found that head and neck cancer cells treated with small RNA molecules that silence a gene called UROD are unusually susceptible to ionizing radiation. UROD encodes an enzyme involved in producing iron-containing haem molecules, and reducing its levels caused oxidative damage and cell death. When implanted into mice that were then treated with radiation, tumour cells with lowered levels of the enzyme grew more slowly than cells containing normal levels.

Furthermore, head and neck tumour samples expressing the lowest levels of $U R O D$ tended to come from patients who responded well to treatment. Drugs that inhibit UROD could make radiation treatments more effective, the authors say. Sci. Transl. Med. 3, 67ra7 (2011)

\section{METAGENOMICS}

\section{Cow spills guts for biofuels}

A dearth of ways to efficiently digest the plant fibre cellulose has stymied efforts to develop plant-based biofuels. Genomic

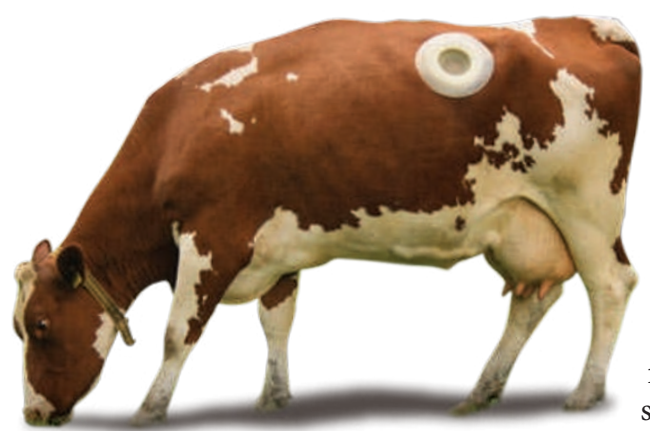
carbohydrate digestion,

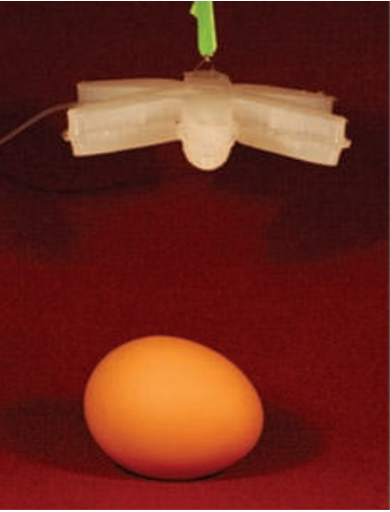
were collected directly from the cow rumen through a port (pictured). The researchers identified 27,755 genes possibly involved in and produced protein from 90 that had similarities to known

sequencing of bacteria from the cow's digestive system has a team of the Lawrence Berkeley

National Laboratory in Berkeley, California.

The researchers sequenced microbes that adhered to plan material in the rumen of the cow, a champion cellulose digester. The microbes 2011
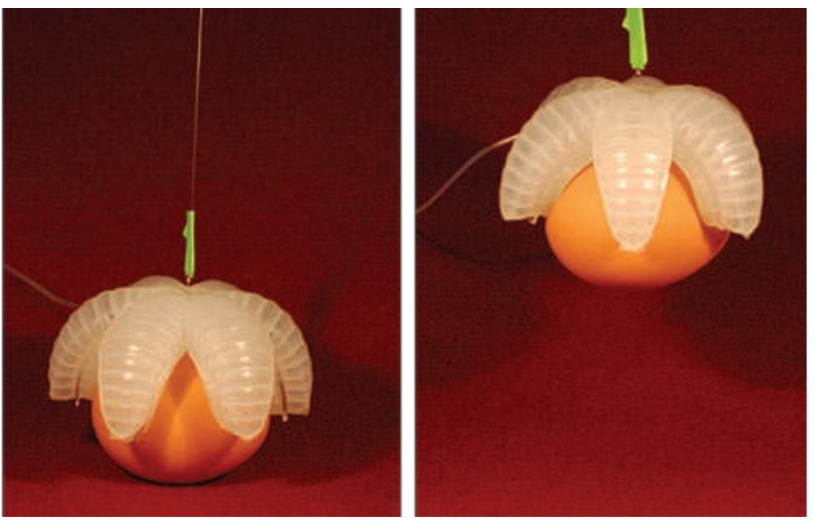

\section{MATERIALS}

\section{'Soft' robot has deft touch}

Robots made from hard materials are not well equipped to handle fragile objects, so researchers have created prototypical 'soft' bots from elastic polymers. These can eggs.

George Whitesides and his colleagues at Harvard University in Cambridge, Massachusetts, embedded balloon-like channels in moulded silicones to create materials that change shape in specific ways when air is pumped into the channels. This can generate complex motion from a single source of pressure, leading to designs such as the team's starfish-shaped gripper (pictured).

With appropriate materials, this technology might eventually produce robots that can handle heavy loads or conduct electricity. Angew. Chem. Int. Edn doi:10.1002/anie.201006464 (2011) enzymes with particular attributes. More than half demonstrated enzymatic activity against a panel of 10 cellulose-containing plants. Science 331, 463-467 (2011)

\section{Omega-3s affect brain signalling}

A diet low in omega-3 fatty acids - typically found in fish oils - has been associated with mood disorders. To find a molecular link, Sophie Layé at the University of Bordeaux in France, Olivier Manzoni at the French National Institute of Health and Medical Research in Marseilles and their co-workers fed mice a diet that was either high or low in omega-3s. They then looked at the ability of neurons in the prefrontal cortex, a brain region thought to mediate emotional behaviour, to alter the strength of their connections - a process known as synaptic plasticity. The authors focused on lipid signalling molecules called endocannabinoids and their receptors, which are involved in this process.

They found that mice whose diets were low in omega- $3 \mathrm{~s}$ had lower levels of the fats, and reduced synaptic plasticity, in the prefrontal cortex. This was due to the decoupling of the cannabinoid receptors from certain proteins that normally bind to them. Mice fed the low-omega-3 diet also showed behavioural signs of depression and anxiety.

Nature Neurosci. doi:10.1038/ nn.2736 (2011) 